\title{
Survey of the Home Music Environment of Children with Various Developmental Profiles
}

\author{
Selena Steinberga ${ }^{\mathrm{a}}$, Carolyn M. Shivers ${ }^{\mathrm{b}}$, Talia Liu ${ }^{\mathrm{c}}$, Laura K. Cirelli ${ }^{\mathrm{d}}$, Miriam D. Lense
}

${ }^{a}$ Department of Psychology and Human Development, Vanderbilt University, 230 Appleton

Place, Nashville, Tennessee 37203, USA, selenasteinberg@gmail.com

${ }^{b}$ Department of Human Development and Family Science, Virginia Polytechnic Institute and

State University, 295 West Campus Drive, Blacksburg, Virginia 24061, USA, shivercm@vt.edu

${ }^{c}$ Department of Otolaryngology-Head and Neck Surgery, Vanderbilt University Medical Center,

$121521^{\text {st }}$ Avenue South, Nashville, Tennessee 37232,USA, talia.liu@vumc.org;

miriam.lense@vanderbilt.edu

${ }^{d}$ Department of Psychology, University of Toronto Scarborough, 1265 Military Trail, Toronto,

Ontario,M1C1A4, Canada, laura.cirelli@utoronto.ca

Declarations of Interest: none

Correspondence concerning this article should be addressed to Miriam Lense, Department of

Otolaryngology-Head and Neck Surgery, 1215 21st Avenue South, Nashville, TN 37232, Email:

miriam.lense@vanderbilt.edu, Phone: 615 322-3086

This article is in press at Journal of Applied Developmental Psychology 


\title{
Survey of the Home Music Environment of Children with Various Developmental Profiles
}

\author{
Abstract \\ Families with young children with and without developmental disabilities often create a \\ musically rich home environment. Parent-child music engagement, like singing play songs, is \\ associated with positive outcomes for children, parents, and their relationship. However, little is \\ known about if the home music environment differs across diagnostic groups and if parent-child \\ music engagement relates to parent-child affective attachment across families of diagnostically \\ diverse children. Using an online questionnaire, the current study examined the home music \\ environment of 340 families with young children with typical and atypical development. A \\ variety of musical activities were common in all diagnostic groups. Diagnostic groups differed in \\ active musical engagement, potentially relating to the differing phenotypes of various \\ developmental disabilities. Parent-child music engagement was associated with parent-child \\ affective attachment, even when controlling for relevant variables. Promoting musical \\ engagement at home and through parent-child therapy may be an accessible way to support \\ parent-child relationships.
}

Keywords: music, parent-child attachment, autism, Williams syndrome, Down syndrome 


\section{Survey of the Home Music Environment of Children with Various Developmental Profiles}

“My son absolutely loves listening, dancing, and 'singing' to songs. It's our favorite way to calm him down if he's upset, and it's our go-to activity when we need something to do." - parent of a 14-month-old child with typical development

"Singing songs and playing musical games makes my daughter light up, even more so when those around her engage." - parent of a two-year-old child with Down syndrome

Everyday parent-child musical activities, like playing musical games and singing lullabies, are common in early childhood (Politimou et al., 2018). A growing body of research suggests that parent-child musical engagement (active participation in musical activities) may support dyadic attachment by promoting joint attention and emotional connection between parent and child (Creighton et al., 2013; de l'Etoile, 2006; Fancourt \& Perkins, 2018; O'Gorman, 2006; Perkins et al., 2018; Persico et al., 2017). Strong parent affective attachment to their child, i.e., the emotional connection a parent has toward their child and the parent-child relationship (Condon \& Corkindale, 1998), is important for parents' and children's well-being (de Cock et al., 2016; de Cock et al., 2017; Johnson, 2013).

In addition to evidence from families of children with typical development (TD), a growing literature in the field of music therapy suggests that beneficial effects of parent-child musical engagement may also extend to families of children with developmental disabilities (O’Donoghue, 2017; Thompson et al., 2014, 2017). The behavioral phenotypes and developmental delays associated with different disabilities may impact parent-child interactions and relationships (Spiker et al., 2002). However, despite the ubiquity of musical activities in 
parent-child play (Levitin et al., 2004; Politimou et al., 2018), little is known about whether the home music environment, including parent-child musical engagement, differs for families of children with atypical development. Such information would inform if and how a common form of parent-child interaction is impacted by different developmental profiles, as well as the potential importance of musical engagement as a component of the parent-child relationship across families of diagnostically diverse children. In the current study, we use an online survey to examine the parent-reported home music environment of young children with and without developmental disabilities and the relationship between parent-child musical engagement and parent-child affective attachment.

\section{Home Music Environment of Families of Children with TD}

Much of the current research on the home music environment centers around families of children with TD. Parents incorporate music into the lives of their young children on a regular basis (Blackburn, 2017; Custodero, et al., 2003; Mendoza \& Fausey, 2019; Williams et al., 2015). A survey study of over 2,000 parents with children under three years of age in the United States reported that $60 \%$ of parents sang or played music to their child daily while $92 \%$ did so on a weekly basis (Custodero et al., 2003). Mendoza et al. (2019) analyzed daylong audio recordings of infants and found that music exposure made up about $9 \%$ of their day. Recorded music (including both instruments and vocals) was more common than live music (e.g., caregiver singing). Reports on the frequency of home musical activities in older preschool-aged children are mixed with some studies suggesting daily home music use (Blackburn, 2017) and other studies reporting that music use at home is less common (de Vries, 2009). These discrepancies may in part be due to how different studies define musical activities, as well as parents' interpretations of what counts as a musical activity. For example, studies may differ regarding 
whether they include only shared parent-child activities versus child-only activities, if they involve active musical activities (e.g., singing, dancing) and/or passive musical activities (e.g., listening to a $\mathrm{CD}$ or the radio in the car), or if the use of musical toys and apps/videos or incorporation of musical instruments are counted.

\section{Benefits of Musical Engagement for Children and Parents}

Musical engagement is associated with a variety of positive social and emotional outcomes for both children and parents, and parent-child musical games appear to serve as an effective medium for sharing social attention (Lense \& Camarata, 2020). Maternal singing captures and maintains infants' attention and modulates their arousal level (de l'Etoile, 2006;

Trehub et al., 2016; Cirelli et al., 2020a; Cirelli \& Trehub, 2020; Nakata \& Trehub, 2004). Infants show preferences for and prosocial behavior toward people that sing the same songs as their parents (Cirelli \& Trehub, 2018; Mehr et al., 2016). Shared musical engagement is also associated with prosocial behavior like cooperation in preschool-aged children (Cirelli et al., 2020b; Kirschner \& Tomasello, 2010). Specifically, in a longitudinal study, younger and older siblings' rates of prosociality positively correlated with older siblings' musical play (Cirelli et al., 2020b), and four-year-old children who engaged in joint music making subsequently demonstrated increased peer helpfulness compared to a control group who engaged in nonmusical play (Kirchner \& Tomasello, 2010). The frequency of shared musical activities between preschoolers and their parents, including playing music, singing songs, and dancing, significantly predicts children's prosocial skills two years later (Williams et al., 2015).

Parent-child musical engagement also benefits caregivers, though most prior research focuses on parents of children in the first year of life. Maternal singing to infants modulates parents' arousal levels (Cirelli et al., 2020a) and mothers smile more when singing (versus 
speaking) to their infant (Trehub et al., 2016). Cross-sectional studies reveal that mothers' singing to their infant is associated with feelings of closeness to their child (Creighton et al., 2013) and reduced maternal depressive symptoms (Custodero et al., 2003). In a large crosssectional survey study with parents of children 0-3 years of age, greater levels of parental singing/musical play were correlated with reduced parental frustration with their child and faster parental responsivity to child crying (Custodero et al., 2003). Such correlational designs are supported by experimental studies in which mother-infant dyads participate in singing or nonmusical interactions: mothers' singing leads to increased feelings of maternal closeness to their child (Fancourt \& Perkins, 2018), increased positive affect (Fancourt \& Perkins, 2018), reduced depressive symptoms (Fancourt \& Perkins, 2017), and increased maternal well-being and selfesteem (Fancourt \& Perkins, 2017). Mothers participating in a music and movement program with their infant reported greater affective attachment to their child and exhibited greater affective engagement during a play interaction than mothers who did not participate in the program (Vlismas et al., 2013).

\section{Musical Interests of Children with Developmental Disabilities}

Musical interests are commonly reported in individuals with developmental disabilities such as Williams syndrome (WS), Down syndrome (DS), and Autism spectrum disorder (ASD) (Lense \& Dykens, 2011; Levitin et al., 2004; Rosner et al., 2004; Sellinger et al., 2006; Thakur et al., 2018). ASD is a common (1 in 54 children) neurodevelopmental disorder characterized by impairments in social engagement and communication (American Psychiatric Association, 2013; Centers for Disease Control and Prevention, 2020). DS (1 in 700) and WS (1 in 10,000) are rare genetic disorders associated with high interest in interacting with others, although difficulties with social pragmatics are also common (Dykens \& Rosner, 1999; Rosner et al., 2004). 
Engaging in musical activities such as singing, dancing, listening to music, or playing an instrument are reported to be positive experiences for children with these developmental disabilities (e.g., Dickie et al., 2009; Levitin et al., 2004; Rosner et al., 2004). In general, musical skills in these populations are frequently observed at levels commensurate with either chronological age expectations (e.g., ASD; Jamey et al., 2019) or mental age expectations (e.g., WS; Don et al., 1999). High levels of emotional responsiveness to music are reported in WS, ASD, and DS (Allen et al. 2013, Lense \& Dykens, 2011; Lense et al., 2013; Levitin et al., 2004) including links between music and social-emotional skills in WS (Lense et al., 2013; Lense \& Dykens, 2016). Intact responses to musical emotions, despite impairments with non-musical social emotions, are observed in ASD (Allen et al., 2009; Allen et al., 2013; Gebauer et al., 2014).

\section{Home Music Environment for Families with Children with Developmental Disabilities}

Prior studies from fields such as music therapy and early childhood/developmental psychology have examined the home music environment broadly, and parent-child musical engagement specifically, in families of young children with developmental disabilities.

Consistent with research with TD infants, infant-directed singing maintains the attention of infants with DS and ASD (de 1'Etoile, 2015; Simpson et al., 2013). Mothers of children with DS take more time to establish a rhythmic structure when singing than mothers of TD infants, perhaps due in part to the child's developmental skills (e.g., slower processing time) as well as added time needed for the parent to read and respond to the child's cues (de l'Etoile et al., 2017). Parents of preschoolers with ASD increase their physical responsiveness to their child (e.g., by imitating or assisting child's play actions) during musical versus non-musical play (Boorom et al., 2020). Preschoolers with ASD were more attentive during a book sharing activity and parents 
looked at their child more when the dyad interacted with song versus picture books (Liu et al., 2019). Parents of young children with ASD report that musical play, compared to non-musical block play, provides an opportunity for shared enjoyment and parent-child interaction (Thompson et al., 2019). Families of children with various developmental disabilities participating in family-centered music therapy report that music activities provide an enjoyable shared experience for both the parent and child (O’Donoghue, 2017; Thompson et al., 2014, 2017). Parent-child music therapy groups also report effects on parenting behaviors such as reduced levels of irritability towards, increased engagement with, and increased acceptance of their child, and improvements in parent mental health (Nicholson et al., 2008). Musical activities appear to be of interest to and provide a context for shared parent-child engagement across families of young children with and without developmental disabilities (Lense \& Camarata, 2020). However, clarification is needed about the range of specific musical activities and if they differ across families of children with various developmental profiles.

\section{Music and Parent-Child Affective Attachment}

Whether parent-child music engagement relates to parent-child affective attachment has not been directly addressed in a large sample that considers children's diagnostic status. The behavioral phenotypes associated with different developmental disabilities may impact parentchild interactions and play. For example, interest and motivation to interact with others is greater in children with WS and DS than in children with ASD (Jones et al., 2000). At the same time, children with ASD, WS, and DS may exhibit impairments in social attention, communication, and emotion regulation and unclear play behaviors, all of which may impact parents' responsiveness and affective attachment to their child (Baptista et al., 2018; Goodman \& Glenwick, 2012; Spiker et al., 2002). Also, compared to parents of children with TD, parents of 
children with developmental disabilities often have elevated levels of parent stress and mental health concerns (Ashworth et al., 2019; Keenan et al., 2016; Pastor-Cerezuela et al., 2020). This is particularly observed in parents of children with ASD and may relate to children's social and behavioral challenges (Eisenhower et al., 2005; Pastor-Cerezuala et al., 2020). High parenting stress and internalizing problems are associated with low parent-child affective attachment in families of children with and without developmental disabilities (de Cock et al., 2016; Dennis et al., 2018; Goodman \& Glenwick, 2012; Willinger et al., 2005). Music therapy interventions with parent-child dyads of children with and without developmental disabilities support parent-child affective attachment including parents' sense of connection to and interactions with their child (e.g., Edwards, 2011; Lindstrøm et al., 2016; Nicholson et al., 2008). If patterns of musical engagement and their relationship with parent-child affective attachment are similar across families of children with and without developmental disabilities, musical engagement may serve common functions across diverse developmental conditions. If patterns and relationships differ, this may inform how diagnostic phenotypes impact common parent-child activities with implications for mechanisms underlying effective use of music, as well as how music is used both informally and for therapeutic purposes in specific disorders.

\section{Current Study}

In the current study, we utilized a parent-report online survey to better understand the home music environment of young children with and without developmental disabilities, including the relationship between parent-child music engagement and parents' affective attachment to their child. The study had two key aims. First, we described the home music environment of families of young children with and without specific diagnoses. We considered three facets of the home musical environment: (1) parent-child shared music engagement; (2) the 
type and amount of specific musical activities in which children participated; and (3) the functions of musical activities in the home. Second, we examined the association between parent-child music engagement and parent-child affective attachment while controlling for relevant demographic and other parenting/family characteristics. We hypothesized that higher levels of parent-child music engagement would be associated with stronger parent-child affective attachment across disability groups.

\section{Method}

\section{Participants}

Participants were 340 caregivers of young children between six months and five years of age with and without developmental disabilities. The respondents were primarily biological mothers $(92.4 \%, n=314)$ and were highly educated (52.3\% had a master's degree or higher). The children included 208 without a diagnosed disability (TD), 39 with ASD, 40 with DS, 25 with WS, and 28 with another disability (OtherDD; e.g., developmental delay, Angelman syndrome, cerebral palsy). The average age of the children was 36.46 months $(S D=17.61)$, the sample was evenly split by sex (50.0\% male), and $37.4 \%$ of the children were the only child in their family. All ages were well-represented across the wide age range. Full demographics by diagnostic group can be found in Table 1.

\section{Procedure}

Data were collected via an online survey using REDCap, a secure, online data server (Harris et al., 2009). We used an online survey in order to be able to reach a large number of families including families of children with rare developmental disabilities. The survey was advertised through social media and various national, state, and local child and disability service organizations in the United States and Canada, including DS-Connect ${ }^{\circledR}$ and the Williams 
Syndrome Association. The survey took 20-30 minutes to complete and was available online from March 2019 to March 2020. This study was approved by the university IRB. Participants provided informed consent prior to completing the survey.

\section{Measures}

\section{Home Music Environment}

Parent-Child Music Engagement. Parents reported parent-child engagement with music using an adapted version of the Music@Home scale (Politimou et al., 2018). The Music@Home scale is a validated and reliable measure of the home musical environment of families with children under five years of age. While the original Music@Home scale includes four subscales plus a total scale, we included a subset of 17 items from subscales that reflected parent and child active engagement with music. The 17 items were summed for a Music@Home total score $(\alpha=$ $.91)$ and were also divided into two subscales: Parent Initiation of Music Activities $(\alpha=0.91)$ and Child's Active Engagement with Music $(\alpha=.92)$. Parents responded using a 7-point Likert scale ranging from "completely disagree" to "completely agree" to indicate their agreement with statements such as "I sing to/with my child in many different situations (e.g., during playtime, with friends or family)" (Parent Initiation subscale) and "I have noticed my child moving in time with the beat of the music" (Child's Active Engagement subscale).

Frequency and Types of Specific Child Music Activities. Parents reported the average time their children spent each day in seven music activities (singing, playing instruments, listening to recorded music, playing with musical toys, watching music videos on a TV/tablet, dancing to music, and engaging in music activities with another person) on a 7-point scale ranging from " 0 minutes" to "More than 2 hours." Scores were averaged across all items for one score indicating mean time spent in each activity (possible range 0-6), and individual items were 
examined to investigate specific musical activities. Reliability for averaging across items was adequate $(\alpha=.78)$.

Functions of Musical Activities. Parents reported the reasons why they and other family members use music with their child for ten possible functions of musical activities (e.g., to soothe the child when upset, to get child's attention, as part of night-time routines, to practice communication skills). Parents used a 7-point Likert scale, ranging from "completely disagree" to "completely agree" to indicate if they used music for each function. For analysis, responses for each item were re-coded into either disagree or agree and then summed for a total number of functions of musical activities (possible range $0-10$ ).

\section{Parent-Child Affective Attachment}

Parent report of affective attachment to their child was assessed with the Postnatal Attachment Questionnaire (PAQ; Condon \& Corkindale, 1998). This validated 19-item measure assesses parents' affective attachment to their children using Likert scales. Although the scale was initially developed for use with infants, it is considered appropriate for use by parents of older children due to its emphasis on parents' feelings of affective attachment toward their child (and not children's attachment to their parent). Consistent with prior studies that used this scale with parents of older children, including children with developmental disabilities (e.g., Goodman \& Glenwick, 2012; Steinberg et al., 2021), we replaced the word "baby" with the word "child." Reliability in the current sample was adequate $(\alpha=.78)$.

\section{Non-Musical Family Activities}

To control for overall time spent engaging in parent-child activities, parents completed a Family Activities Questionnaire. The questionnaire was adapted from questions used in the Millennium Cohort Study (Hansen et al., 2010) and is similar to surveys used in other studies 
assessing families' musical and non-musical activities (e.g., Nicholson et al., 2010). Parents reported how frequently they engaged in six different activities with their child (e.g., reading, drawing, playing outdoor games) on a 5-point scale from "hardly ever" to "several times a day $(>3 x)$." Although the scale initially included an item for "Playing music, singing songs, other musical activities," that item was not included in the total, resulting in total possible scores ranging from 5-25 reflecting Non-Musical Family Activities $(\alpha=.67)$.

\section{Parent Distress}

Parent psychological distress was assessed with the Kessler Screening Scale for Psychological Distress (K6), a 6-item scale of mental health symptoms related to depression and anxiety (Kessler et al., 2002). The K6 is widely used as part of national health screeners (Kessler et al., 2010; Slade et al., 2011). Parents reported how often they felt each of the six symptoms over the past four weeks on a 5-point Likert scale from "none of the time" to "all of the time" and items were summed for a total parent distress score $(\alpha=.82)$.

\section{Parent Self-Efficacy}

Parent self-efficacy was measured with a single item: "Which of the following statements best describes how you feel about yourself as a parent?" The five response choices ranged from "Not very good at being a parent" to "A very good parent." This item was adapted from the Longitudinal Study of Australian Children (Zubrick et al., 2008).

\section{Data Analysis}

For the first research aim, descriptive statistics for the relevant variables of interest regarding the home music environment were examined (parent-child music engagement [Music@Home]; frequency and types of child music activities; functions of musical activities) and compared across diagnostic groups using MANCOVAs, controlling for child age and parent 
education. To determine the relationship between parent-child music engagement and parents' affective attachment to their child (the second research aim), bivariate correlations were run among parent-child music engagement (Music@Home total), parent-child affective attachment, and relevant demographic and parenting control variables (parent self-efficacy, parent distress, child age, and non-musical family activities). A univariate ANCOVA was used to test the relationship among parent-child music engagement (Music@Home), parent-child affective attachment, and diagnosis while controlling for demographic and parenting variables (child age, parent distress, parent self-efficacy, and total non-musical family activities). As is often the case in online questionnaire studies, there was some attrition in participant completion of measures throughout the survey (i.e., incomplete responses). The ANCOVA model examining parent-child attachment included a reduced sample with only participants who completed all measures (total $n$ $=291$ [TD: $n=180$; ASD: $n=35$; WS: $n=21$; DS: $n=31$; OtherDD: $n=24]$ ). Due to the large number of analyses, only results with $p$ values $<.01$ are reported as significant. Effect sizes were interpreted in line with standard conventions (Cohen, 1988): Small, medium, and large effects are conventionally defined as $r$ values of $.1, .3$, and .5 , respectively, and as $\eta^{2}$ values of $.01, .06$, and .14, respectively. Analyses were conducted using SPSS v.26.

\section{Results}

\section{Home Music Environment by Diagnostic Group}

\section{Parent-Child Music Engagement}

Music@Home total scores indicated generally high levels of parent-child music engagement. Music@Home total scores differed by diagnostic group, $F(4,297)=4.273, p<.01$, $\eta^{2}=.054$, with parents of children with DS reporting greater parent-child music engagement than parents of children with ASD or OtherDD (but not WS or TD), and parents of OtherDD children 
reporting less parent-child music engagement than all other groups except parents of children with ASD (Table 2). We then examined the two Music@Home subscale scores. Diagnostic groups did not differ in their Parent Initiation of Music Activities scores but did differ in their Children's Active Engagement with Music scores, $F(4,297)=10.491, p<.001$. Children with ASD had lower levels of Active Engagement with Music than children with DS, WS, or TD, and children with OtherDD had lower levels of Active Engagement with Music than children with DS or TD when controlling for child age (Table 2).

\section{Frequency and Type of Specific Child Music Activities}

There were no diagnostic group differences in overall child music activity time (i.e., mean time spent in each music activity). Scores indicate that children generally spent between 10 and 29 minutes a day in each musical activity. When examining specific types of musical activities, diagnostic groups differed in how much time children spent dancing to music, $F(4$, $298)=9.79, p<.01, \eta^{2}=.060$, and singing, $F(4,298)=10.81, p<.01, \eta^{2}=.050$. Specifically, parents of children with ASD and OtherDD reported their children spend less time dancing than parents of children with DS $(p<.01)$. Parents of children with OtherDD reported that their children spend less time singing than parents of children with WS or TD $(p<.01$ for all). There were no other differences among individual items.

\section{Functions of Music}

Diagnostic groups did not differ in the total number of functions of music they reported using. Across all participants, parents reported using music for an average of $7.8(S D=2.2)$ reasons. All ten functions of music were endorsed by over $50 \%$ of participants in each diagnostic group (Table 3). The most frequently endorsed functions of music were to keep busy or pass the 
time while waiting (92.1\% of participants), to interact in social games (87.7\% of participants), and to soothe their children when upset (83.2\% of participants).

\section{Correlations with Parent-Child Affective Attachment}

Parent-child affective attachment differed by diagnostic group with highest scores in the DS and TD groups and lower scores in the ASD and OtherDD groups (Table 2). Parent-child affective attachment was significantly correlated with the parenting variables including a large positive correlation with parent self-efficacy, $r=.52, p<.001$, and a large negative correlation with parent distress, $r=-.50, p<.001$. Consistent with prior research, stronger parent-child affective attachment was associated with greater parent sense of own parenting skill and less mental health distress (Atkinson, 2000; Kohlhoff \& Barnett, 2013). Parent-child music engagement (Music@Home total score) had a small-to-medium correlation with parent-child affective attachment, $r=.35, p<.001$. All correlation coefficients can be found in Table 4 .

\section{Model Examining Parent-Child Affective Attachment}

The final ANCOVA model examining parent-child affective attachment was a significant fit, $F(10,278)=30.036, p<.001, \eta_{\mathrm{p}}^{2}=.519$, adjusted $R^{2}=.502$. Parent distress, parent selfefficacy, respondent education, and the Music@Home total score all independently related to parent-child affective attachment and were significant at the $p<0.001$ level. Effect sizes were large for parent distress $\left(\eta_{\mathrm{p}}^{2}=.143\right)$ and parent self-efficacy $\left(\eta_{\mathrm{p}}{ }^{2}=.205\right)$, medium for respondent education $\left(\eta_{\mathrm{p}}{ }^{2}=.089\right)$ and small-to-medium for parent-child music engagement $\left(\eta_{\mathrm{p}}{ }^{2}=.041\right)$. The effect size was also small-to-medium for child diagnosis $\left(\eta_{\mathrm{p}}^{2}=.041\right)$ though this result did not reach our significance threshold $(p=.021)$. The full coefficients for the model can be found in Table 5 .

\section{Discussion}


The current survey study demonstrates that musical activities are common among families of young children with and without developmental disabilities with both similarities and differences across diagnostic groups. Across all diagnostic groups, caregivers reported parentchild shared music engagement, that their children engage in various types of musical activities, and that families use music for a variety of functions. Parent-child music engagement was associated with parent-child affective attachment, even when controlling for other important parenting characteristics, though it is important to note that directionality of this relationship cannot be determined in a correlational study. Regardless, engagement with music in families of children with varying developmental profiles highlights music as a natural and accessible activity for parent-child interactions (Lense \& Camarata, 2020).

When examining the home music environment by diagnostic group, there was some evidence for greater musical engagement in children with DS or WS compared to children in the ASD and OtherDD groups for specific types of activities (though note that only the ASD and OtherDD groups significantly differed from the TD group when using a $p<.01$ level for significance). Across both the Music@Home scale and the frequency and types of specific child music activities surveys, diagnostic group differences were observed for music activities in which the child has an active, physical role, such as dancing or singing. The reduced participation in movement activities to music in some diagnostic groups may relate to motor and speech impairments, as well as auditory-motor integration abilities, which are important for moving to music (e.g., Grahn \& Brett, 2007). The OtherDD group included children with a variety of disorders including disorders with significant speech and motor deficits (e.g., Angelman syndrome [Williams et al., 2010]) or motor impairments (e.g., cerebral palsy), and non-syndromic developmental delay (which can include speech and motor delays). Many 
children with ASD also exhibit speech and motor difficulties, including challenges with motor coordination and auditory-motor integration (Isenhower et al., 2012; Kauer et al., 2018; Sharda et al., 2018). At the same time, motor and speech delays are also common in young children with WS and DS (Kent \& Vorperian, 2013; Mervis et al., 2011), suggesting that impairments in these domains may not fully account for the diagnostic differences in these types of musical activities. Different profiles for children's active engagement with music may also relate to the social phenotype of the different disorders. Singing and moving to music often occur as part of social interactions and children may imitate these behaviors when they are modelled by their social partners (e.g., Cirelli \& Trehub, 2019; Trehub \& Gudmundsdottir, 2019). Moving synchronously with others to music is associated with subsequent social behavior such as helping in young TD children (Cirelli et al., 2014; Kirschner \& Tomasello, 2010). ASD is characterized by reductions in social engagement (American Psychiatric Association, 2013) including reduced interpersonal movement synchrony across a variety of tasks (McNaughton \& Redcay, 2020). In contrast, individuals with disorders such as WS and DS generally exhibit high social motivation to interact with others (Dykens \& Rosner, 1999; Rosner et al., 2004). Musical rhythm skills and movement to music have been associated with social skills in clinical populations including adults with WS (Lense \& Dykens, 2016) and young children with ASD (Lense, 2018). In a prior cross-sectional study of Music@Home with TD infants, children's active engagement with music (but not parent initiation of music), was correlated with parent-child shared reading and play activities (Politimou et al., 2018). Thus, active music engagement activities may be related to children's interest in engaging with others, though directionality between these domains cannot be determined in correlational studies. Children who are more interested in engaging with others in activities may also be more interested in active musical engagement; and/or higher 
active musical engagement (perhaps promoted by a parent) may facilitate a child in engaging with others. Future longitudinal and experimental research involving direct assessment of musical engagement and children's developmental skills across a variety of domains, including cognitive, motor, and social areas, over time can further examine the relationship and directionality between these skill areas in children with TD and different developmental disabilities.

Adding to prior research investigating types of music use in the home (e.g., Mendoza \& Fausey, 2019) or parents' perceived benefits of music use for their child (e.g., to aid in numeracy/literacy skills, [de Vries, 2009]), this study captures music's most common functions in the home environment. Across all diagnostic groups, the most common functions were to keep busy or pass the time while waiting, to interact in social games, and to soothe the child when upset. The high parent endorsement of music use in between activities, during play time, and during child negative states suggests that parents naturally and successfully utilize music in a variety of situations to modulate children's affect and engagement.

Across all diagnostic groups, parent-child music engagement was related to parent-child affective attachment even when controlling for other highly significant correlates of parent affective attachment such as parent distress, parenting efficacy, and parent education. This relationship appeared to be specific to musical activities, as non-musical family activities were not associated with affective attachment when controlling for these parenting and demographic variables. Greater parenting stress and anxiety are frequently observed in parents of children with specific developmental disabilities, and as evident in the current study and prior literature, both stress and parenting efficacy relate to parent-child attachment (Ashworth et al., 2019; Goodman \& Glenwick, 2012; Keenan et al., 2016; Phillips et al., 2017). Yet, even when considering these 
important contributors to parent-child affective attachment, the current study suggests that either families with positive parent-child relationships create a more musical home environment, and/or that music may be a viable medium for supporting the parent-child relationship across developmentally diverse children and families. Accordingly, musical contexts and therapies (e.g., parent-mediated music interventions; family-centered music therapy) are increasingly used to support parent-child interactions in families at-risk due to child developmental concerns and/or parent mental health problems (Fancourt \& Perkins, 2018; Hernandez-Ruiz, 2019; Lense \& Camarata, 2020; Perkins et al., 2018; Teggelove et al., 2019).

There are multiple avenues through which parent-child musical engagement may impact parent-child affective attachment. Musical activities may facilitate positive emotional states for both parents and children (Cirelli et al. 2020; Creighton et al., 2013; Vlismas et al., 2013). If parents are achieving their intended goal through use of music (e.g., soothing their child), musical activities may increase parents' sense of parenting efficacy (Lense et al., 2019). The affordances of musical activities may also help parents engage in shared or reciprocal play with their children (e.g., joint singing or dancing, turn-taking or imitation games in song), which may enhance parent responsiveness (Hernandez-Ruiz, 2019; Lense \& Camarata, 2020). These potential mechanisms are supported by parents' free responses on the current survey, in which many parents reported positive shared emotions and experiences with their children during music-making activities. Sharing musical games together with her child with TD made a parent feel "like we have something in common", while a parent of a child with DS claimed, “spontaneous family dance time always feels like a bonding experience." A parent described her reaction to her child with ASD's musical play as "he dances and I feel so much joy seeing him so happy." Future longitudinal and intervention research should investigate specific pathways that 
mediate the relationship between music engagement and parent-child attachment in TD and different disability groups.

Strengths of the current study include the inclusion of children with and without developmental disabilities particularly in specific diagnostic groups, the use of a validated parent-child music engagement questionnaire, and the diversity of specific information about musical activities. Our larger age range extends prior findings on music and parent-child attachment in families of TD infants (e.g., Perkins et al., 2018; Vlismas et al., 2013) to the preschool years. Additionally, we examined the relationship between parent-child music engagement and affective attachment when controlling for important parent variables of mental health, distress, and parenting efficacy.

However, there are also several limitations to be addressed in future studies. The majority of participants were well-educated; a sample with more heterogeneity in education level would help to understand musical engagement and generalize findings across a more representative sample of families. Future studies should also collect information on the socio-economic status (beyond parent education) and race/ethnicity of participants in order to better understand the ways that the sample represents the general population. Similarly, participants were primarily biological mothers, but it is also important to understand the relationship between music and other familial relationships (Lense et al., 2020). Future studies could also expand to larger samples of specific disability populations, particularly those in the OtherDD group. While the survey was advertised through a variety of community and disability organizations, it is possible that the parents who took the time to fill out the survey may be particularly interested in using music in the home. 
The current study involved a parent-report survey collected at one time point. Future studies could expand on the current findings by directly assessing parent-child musical and affective attachment behaviors through home-based or laboratory-based observations. For example, studies can directly code child and parent behavioral (e.g., movement/gestures, communication) and physiological (e.g., markers of arousal) levels and coordination during musical activities (e.g., Boorom et al., 2020; Cirelli et al., 2019; Cirelli et al., 2020a; Lense et al., 2020; Liu et al., 2019). As well, parent and child behavior can be measured via assessments and parent questionnaires longitudinally during family participation in music therapy, which would further understanding of parent and child behavior change during and in response to music (Gottfried et al., 2018; Wheeler, 2018). Additionally, due to the cross-sectional nature of the study, causal directionality of the relationship between parent-child music engagement and parent-child attachment cannot be determined. It is unclear whether parent with greater affective attachment to their child also engage in more musical interactions, and/or if families that engage in parent-child musical activities consequently have more positive parent-child affective attachment.

The present study examined 1) home musical activities among families of children with and without developmental disabilities, and 2) how parent-child musical engagement relates to parent-child affective attachment. Results show robust use of music across diagnostic groups, with variability in specific types of active musical engagement for children with different developmental disabilities. Parent-child music engagement was associated with parent-child affective attachment, even when accounting for parent distress and parenting self-efficacy. These findings are consistent with the use of music-based activities to support the parent-child 
relationship among families of children with and without developmental disabilities and can inform the design of future intervention studies using music for these populations.

\section{Acknowledgments}

We have no known conflicts of interest to disclose. Funding Support: Research reported in this publication was supported in part by awards from the National Endowment for the Arts (1844332-38-C-18), as well as the National Institute on Deafness and Other Communication Disorders (R21DC016710), the National Institute of Mental Health (R61MH123029) and National Center for Complementary and Integrative Health (NCCIH) of the National Institutes of Health, and the NCATS/NIH (UL1 TR000445). The content is solely the responsibility of the authors and does not necessarily represent the official views of the National Institutes of Health $(\mathrm{NIH})$ or the National Endowment for the Arts (NEA). The NIH and NEA do not guarantee the accuracy or completeness of the information included in this report and are not responsible for any consequence of its use. This work was additionally supported by the Program for Music, Mind \& Society at Vanderbilt (with funding from the Trans-Institutional Programs Initiative), the VUMC Faculty Research Scholars Program, and the VUMC Department of Otolaryngology. The authors acknowledge the contribution of DS-Connect ${ }^{\circledR}$ (The Down Syndrome Registry) which is supported by the Eunice Kennedy Shriver National Institute of Child Health and Human Development (NICHD), NIH for the study recruitment used in this manuscript. 


\section{References}

Allen, R., Hill, E., \& Heaton, P. (2009). 'Hath charms to soothe...': An exploratory study of how high-functioning adults with ASD experience music. Autism, 13(1), 21-41.

Allen, R., Davis, R., \& Hill, E. (2013). The effects of autism and alexithymia on physiological and verbal responsiveness to music. Journal of Autism and Developmental Disorders, $43(2), 432-444$.

American Psychiatric Association. (2013). Diagnostic and statistical manual of mental disorders (DSM-5®). American Psychiatric Pub.

Ashworth, M., Palikara, O., \& Van Herwegen, J. (2019). Comparing parental stress of children with neurodevelopmental disorders: The case of Williams syndrome, Down syndrome and autism spectrum disorders. Journal of Applied Research in Intellectual Disabilities, 32(5), 1047-1057.

Atkinson, L., Paglia, A., Coolbear, J., Niccols, A., Parker, K. C., \& Guger, S. (2000). Attachment security: A meta-analysis of maternal mental health correlates. Clinical Psychology Review, 20(8), 1019-1040.

Baptista, J., Sampaio, A., Fachada, I., Osório, A., Mesquita, A. R., Garayzabal, E., Duque, F., Oliveira, G., \& Soares, I. (2019). Maternal interactive behaviours in parenting children with Williams Syndrome and Autism Spectrum Disorder: Relations with emotional/behavioural problems. Journal of Autism and Developmental Disorders, 49(1), 216-226. https://doi.org/10.1007/s10803-018-3715-6

Belsky, J., \& Fearon, R. M. P. (2002). Infant-mother attachment security, contextual risk, and early development: A moderational analysis. Development and Psychopathology, 14(2), 293-310. http://dx.doi.org/10.1017/S0954579402002067 
Blackburn, C. (2017). Young children's musical activities in the home. Education 3-13, 45(6), 674-688. http://dx.doi.org/10.1080/03004279.2017.1342320

Boorom, O., Muñoz, V., Xin, R., Watson, M., Lense, M.D. (2020). Parental responsiveness during musical and non-musical engagement in preschoolers with ASD. Research in Autism Spectrum Disorders.

Center for Disease Control and Prevention (2020). Data and statistics on Autism spectrum disorder.

Cirelli, L. K., Einarson, K. M., \& Trainor, L. J. (2014). Interpersonal synchrony increases prosocial behavior in infants. Developmental Science, 17(6), 1003-1011.

Cirelli, L. K., \& Trehub, S. E. (2019). Dancing to Metallica and Dora: Case study of a 19-monthold. Frontiers in Psychology, 10.

Cirelli, L. K., Jurewicz, Z. B., \& Trehub, S. E. (2020a). Effects of maternal singing style on mother-infant arousal and behavior. Journal of Cognitive Neuroscience, 32(7), 1213 1220.

Cirelli, L. K., Peiris, R., Tavassoli, N., Recchia, H., \& Ross, H. (2020b). It takes two to tango: Preschool siblings' musical play and prosociality in the home. Social Development. https://doi.org/10.1111/sode.12439

Cirelli, L. K., \& Trehub, S. E. (2020). Familiar songs reduce infant distress. Developmental Psychology, 56(5), 861-868. https://doi.org/10.1037/dev000091

Cohen, J. (1988). Statistical Power Analysis for the Behavioral Sciences. New York, NY: Routledge Academic. 
Condon, J. T., \& Corkindale, C. J. (1998). The assessment of parent-to-infant attachment: Development of a self-report questionnaire instrument. Journal of Reproductive and Infant Psychology, 16(1), 57-76.

Creighton, A. L., Atherton, M., Kitamura, C., \& Trondalen, G. (2013). Singing play songs and lullabies: Investigating the subjective contributions to maternal attachment constructs. Australian Journal of Music Therapy, 24, 17-44.

Custodero, L. A., Rebello Britto, P., \& Brooks-Gunn, J. (2003). Musical lives: A collective portrait of American parents and their young children. Journal of Applied Developmental Psychology, 24(5), 553-572. https://doi.org/10.1016/j.appdev.2003.08.005

de Cock, Evi S.A., Henrichs, J., Vreeswijk, C. M. J. M., Maas, A. J., Rijk, C. H. A. M., \& van Bakel, Hedwig J.A. (2016). Continuous feelings of love? The parental bond from pregnancy to toddlerhood. Journal of Family Psychology, 30(1), 125-134.

De Cock, E. S., Henrichs, J., Klimstra, T. A., Maas, A. J. B., Vreeswijk, C. M., Meeus, W. H., \& van Bakel, H. J. (2017). Longitudinal associations between parental bonding, parenting stress, and executive functioning in toddlerhood. Journal of Child and Family Studies, 26(6), 1723-1733.

de l'Etoile, S. K. (2006). Infant behavioral responses to infant-directed singing and other maternal interactions. Infant Behavior and Development, 29(3), 456-470. https://doi.org/10.1016/j.infbeh.2006.03.002

de 1'Etoile, S.K. (2015). Self-regulation and infant-directed singing in infants with down syndrome. Journal of Music Therapy, 52(2), 195-220. https://doi.org/10.1093/jmt/thv003 
de l'Etoile, S., Behura, S., \& Zopluoglu, C. (2017). Acoustic parameters of infant-directed singing in mothers of infants with down syndrome. Infant Behavior \& Development, 49, 151-160. http://dx.doi.org/10.1016/j.infbeh.2017.09.00

de Vries, P. (2009). Music at home with the under fives: What is happening? Early Child Development and Care, 179(4), 395-405. https://doi.org/10.1080/03004430802691914

Dennis, M. L., Neece, C. L., \& Fenning, R. M. (2018). Investigating the influence of parenting stress on child behavior problems in children with developmental delay: The role of parent-child relational factors. Advances in Neurodevelopmental Disorders, 2(2), 129141.

Dickie, V. A., Baranek, G. T., Schultz, B., Watson, L. R., McComish, C. S. (2009). Parent reports of sensory experiences of preschool children with and without autism: A qualitative study. American Journal of Occupational Therapy, 63(2), 172-181. https://doi.org/10.5014/ajot.63.2.17.

Don, A. J., Schellenberg, E. G., \& Rourke, B. P. (1999). Music and language skills of children with williams syndrome. Child Neuropsychology, 5(3), 154-170. http://dx.doi.org/10.1076/chin.5.3.154.7337

Dykens, E. M., \& Rosner, B. A. (1999). Refining behavioral phenotypes: Personalitymotivation in Williams and Prader-Willi syndromes. American Journal on Mental Retardation, 104(2), 158-169.

Edwards, J. (2011). Music therapy and parent infant bonding. In Edwards, J. (Ed.), Music therapy and parent infant bonding, (5-21). Oxford University Press. 
Eisenhower, A. S., Baker, B. L., \& Blacher, J. (2005). Preschool children with intellectual disability: syndrome specificity, behaviour problems, and maternal well-being. Journal of Intellectual Disability Research, 49(9), 657-671.

Fancourt, D., \& Perkins, R. (2017). Associations between singing to babies and symptoms of postnatal depression, wellbeing, self-esteem and mother-infant bond. Public Health, 145, 149-152.

Fancourt, D., \& Perkins, R. (2018). The effects of mother-infant singing on emotional closeness, affect, anxiety, and stress hormones. Music \& Science, 1 . https://doi.org/10.1177/2059204317745746

Gebauer, L., Skewes, J., Westphael, G., Heaton, P., \& Vuust, P. (2014). Intact brain processing of musical emotions in autism spectrum disorder, but more cognitive load and arousal in happy vs. sad music. Frontiers in Neuroscience, 8.

Goodman, S. J., \& Glenwick, D. S. (2012). Correlates of attachment perceptions in parents of children with Autism Spectrum Disorders. Journal of Autism and Developmental Disorders, 42(10), 2056-2066. https://doi.org/10.1007/s10803-012-1453-8

Gottfried, T., Thompson, G., Elefant, C., \& Gold, C. (2018). Reliability of the music in everyday life (MEL) scale: a parent-report assessment for children on the autism spectrum. Journal of music therapy, 55(2), 133-155.

Grahn, J. A., \& Brett, M. (2007). Rhythm and beat perception in motor areas of the brain. Journal of Cognitive Neuroscience, 19(5), 893-906.

Harris, P. A., Taylor, R., Thielke, R., Payne, J., Gonzalez, N., \& Conde, J. G. (2009). Research electronic data capture (REDCap) — a metadata-driven methodology and workflow 
process for providing translational research informatics support. Journal of biomedical informatics, 42(2), 377-381.

Hansen, K., Jones, E., Joshi, H., \& Budge, D. (2010). Millennium Cohort Study Fourth Survey: A user's guide to initial findings. London: Centre for Longitudinal Studies, University of London.

Hernandez-Ruiz, E. (2019). Feasibility of parent coaching of music interventions for children with Autism Spectrum Disorder. Music Therapy Perspectives. https://doi.org/10.1093/mtp/miz016

Hernandez-Ruiz, E. (2020). Parent coaching of music interventions for children with ASD: A conceptual framework. Nordic Journal of Music Therapy, 29(3), 200-221. https://doi.org/10.1080/08098131.2019.1647447

Isenhower, R. W., Marsh, K. L., Richardson, M. J., Helt, M., Schmidt, R. C., \& Fein, D. (2012). Rhythmic bimanual coordination is impaired in young children with autism spectrum disorder. Research in Autism Spectrum Disorders, 6(1), 25-31.

Jamey, K., Foster, N. E., Sharda, M., Tuerk, C., Nadig, A., \& Hyde, K. L. (2019). Evidence for intact melodic and rhythmic perception in children with Autism Spectrum Disorder. Research in Autism Spectrum Disorders, 64, 1-12.

Johnson, K. (2013). Maternal-infant bonding: A review of literature. International Journal of Childbirth Education, 28(3), 17-22.

Jones, W., Bellugi, U., Lai, Z., Chiles, M., Reilly, J., Lincoln, A., and Adolphs, R. (2000). Hypersociability in Williams syndrome. J. Cognitive Neuroscience. 12(Suppl.), 30-46. 
Kaur, M., Srinivasan, S. M., \& Bhat, A. N. (2018). Comparing motor performance, praxis, coordination, and interpersonal synchrony between children with and without Autism Spectrum Disorder (ASD). Research in Developmental Disabilities, 72, 79-95.

Keenan, B. M., Newman, L. K., Gray, K. M., Rinehart, N. J. (2016). Parents of children with ASD experience more psychological distress, parenting stress, and attachment-related anxiety. Journal of Autism and Developmental Disorders, 46, 2979-2991. https://doi.org/10.1007/s10803-016-2836-z

Kent, R. D., \& Vorperian, H. K. (2013). Speech impairment in Down syndrome: A review. Journal of Speech, Language and Hearing Research (Online), 56(1), 178.

Kessler, R. C., Andrews, G., Colpe, L. J., Hiripi, E., Mroczek, D. K., Normand, S. L., Walters, E. E., \& Zaslavsky, A. M. (2002). Short screening scales to monitor population prevalences and trends in non-specific psychological distress. Psychological Medicine, 32(6), 959976.

Kessler, R. C., Green, J. G., Gruber, M. J., Sampson, N. A., Bromet, E., Cuitan, M., Furukawa T. A., Gureje, O., Hinkov, H., Hu, C., Lara, C., Lee, S., Mneimneh, Z., Myer, L., OakleyBrowne, M., Posada-Viilla, J., Sagar, R., Viana, M. C., \& Zaslavsky, A. M. (2010). Screening for serious mental illness in the general population with the K6 screening scale: results from the WHO World Mental Health (WMH) survey initiative. International Journal of Methods in Psychiatric Research, 19(S1), 4-22.

Kirschner, S., \& Tomasello, M. (2010). Joint music making promotes prosocial behavior in 4year-old children. Evolution and Human Behavior, 31(5), 354-364. https://doi.org/10.1016/j.evolhumbehav.2010.04.004 
Kohlhoff, J., \& Barnett, B. (2013). Parenting self-efficacy: Links with maternal depression, infant behaviour and adult attachment. Early Human Development, 89(4), 249-256.

Lense, M. D. “It Don't Mean a Thing if it Ain't Got that Swing: The Music of Social Interaction.” Invited talk at Mainly Mozart Festival, San Diego, CA, June, 2018.

Lense, M. D., Beck, S., Liu, C., Pfeiffer, R., Diaz, N., Lynch, M., ... \& Fisher, M. H. (2020). Parents, peers, and musical play: Integrated parent-child music class program supports community participation and well-being for families of children with and without autism spectrum disorder. Frontiers in Psychology, 11, 2775.

Lense, M. D., \& Camarata, S. (2020). PRESS-Play: Musical engagement as a motivating platform for social interaction and social play in young children with ASD. Music \& Science, 3 .

Lense, M. D., \& Dykens, E. M. (2011). Musical interests and abilities in individuals with developmental disabilities. International Review of Research in Developmental Disabilities, 41, 265-312. https://doi.org/10.1016/B978-0-12-386495-6.00008-4

Lense, M. D., \& Dykens, E. M. (2016). Beat perception and sociability: Evidence from Williams Syndrome. Frontiers in Psychology, 7. https://doi.org/10.3389/fpsyg.2016.00886

Lense, M. D., Pfeiffer, R., Diaz, N., Lynch, M., Goodman, N., Liu, C., Xin, R., Summers, A., \& Beck, S. (2019, May). Parents, peers, and musical play: A mixed-methods analysis of an integrated parent-child music class program for families of children with and without $A S D$ [Poster presentation]. International Society for Autism Research (INSAR), Montreal, Canada. 
Lense, M. D., Shivers, C., \& Dykens, E. (2013). (A)musicality in Williams syndrome: Examining relationships among auditory perception, musical skill, and emotional responsiveness to music. Frontiers in Psychology, 4, 1-13.

Levitin, D. J., Cole, K., Chiles, M., Lai, Z., Lincoln, A., \& Bellugi, U. (2004). Characterizing the musical phenotype in individuals with Williams syndrome. Child Neuropsychology, $10(4), 223-247$.

Lindstrøm, S., Oscarsson, S., Ridder, H. M., Haslbeck, F., Oldfield, A., Teggelove, K., ... \& Stige, B. (2016). Music therapy with families: Therapeutic approaches and theoretical perspectives. Jessica Kingsley Publishers.

Liu, T., Dai, D., Schultz, B., Liu, C., Lense, M. D. (2019, May). Increasing Parent-Child Social Interactions in Preschoolers with and without ASD through Song Books [Poster presentation]. International Society for Autism Research (INSAR) Annual Meeting, Montreal, Canada.

McNaughton, K. A., \& Redcay, E. (2020). Interpersonal Synchrony in Autism. Current Psychiatry Reports, 22(3), 12.

Mendoza, J. K., \& Fausey, C. M. (2019). Everyday music in infancy. PsyArXiv. https://doi.org/10.31234/osf.io/sqatb

Mervis, C. B., \& Velleman, S. L. (2011). Children with Williams syndrome: language, cognitive, and behavioral characteristics and their implications for intervention. Perspectives on Language Learning and Education, 18(3), 98-107.

Nakata, T., \& Trehub, S. E. (2004). Infants' responsiveness to maternal speech and singing. Infant Behavior and Development, 27(4), 455-464. 
Nicholson, J. M., Berthelsen, D., Abad, V., Williams, K., \& Bradley, J. (2008). Impact of music therapy to promote positive parenting and child development. Journal of Health Psychology, 13(2), 226-238. https://doi.org/10.1177/1359105307086705

Nicholson, J. M., Berthelsen, D., Williams, K. E., \& Abad, V. (2010). National study of an early parenting intervention: Implementation differences on parent and child outcomes. Prevention Science, 11(4), 360-370.

O’Donoghue, J. (2017). The observed experiences of music therapy on parent-child interaction for families with children with Down Syndrome. Voices, 17(2). https://doi.org/10.15845/voices.v17i2.892

O'Gorman, S. (2006). Theoretical interfaces in the acute paediatric context: A psychotherapeutic understanding of the application of infant-directed singing. American Journal of Psychotherapy, 60(3), 271-283.

Pastor-Cerezuela, G., Fernández-Andrés, M. I., Pérez-Molina, D., \& Tijeras-Iborra, A. (2020). Parental stress and resilience in autism spectrum disorder and Down syndrome. Journal of Family Issues.

Perkins, R., Yorke, S., \& Fancourt, D. (2018). How group singing facilitates recovery from the symptoms of postnatal depression: A comparative qualitative study. BMC Psychology, 6(1), 41. https://doi.org/10.1186/s40359-018-0253-0

Persico, G., Antolini, L., Vergani, P., Costantini, W., Nardi, M. T., \& Bellotti, L. (2017). Maternal singing of lullabies during pregnancy and after birth: Effects on mother-infant bonding and on newborns' behaviour. Concurrent Cohort Study. Women and Birth, 30, e214-e220. https://doi.org/10.1016/j.wombi.2017.01.007 
Phillips, B. A., Conners, F., \& Curtner-Smith, M. E. (2017). Parenting children with Down Syndrome: An analysis of parenting styles, parenting dimensions, and parental stress. Research in Developmental Disabilities, 68, 9-19.

Politimou N., Stewart L., Müllensiefen D., Franco F. (2018). Music@Home: A novel instrument to assess the home musical environment in the early years. PLoS ONE, 13(4). https://doi.org/10.1371/journal.pone.0193819

Rosner, B. A., Hodapp, R. M., Fidler, D. J., Sagun, J. N., \& Dykens, E. M. (2004). Social competence in persons with Prader-Willi, Williams and Down's Syndromes. Journal of Applied Research in Intellectual Disabilities, 17(3), 209-217.

Sellinger, M. H., Hodapp, R. M., \& Dykens, E. M. (2006). Leisure activities of individuals with Prader-Willi, Williams, and Down syndromes. Journal of Developmental and Physical Disabilities, 18(1), 59-71.

Sharda, M., Tuerk, C., Chowdhury, R., Jamey, K., Foster, N., Custo-Blanch, M., Tan, M., Nadig, A., \& Hyde, K. (2018). Music improves social communication and auditory-motor connectivity in children with autism. Translational Psychiatry, 8(1), 1-13.

Simpson, K., Keen, D., \& Lamb, J. (2013). The use of music to engage children with autism in a receptive labelling task. Research in Autism Spectrum Disorders, 7(12), 1489-1496.

Slade, T., Grove, R., \& Burgess, P. (2011). Kessler psychological distress scale: normative data from the 2007 Australian National Survey of Mental Health and Wellbeing. Australian \& New Zealand Journal of Psychiatry, 45(4), 308-316.

Slonims, V., \& McConachie, H. (2006). Analysis of mother-infant interaction in infants with Down syndrome and typically developing infants. American Journal on Mental Retardation, 111(4), 273-289. 
Spiker, D., Boyce, G. C., \& Boyce, L. K. (2002). Parent-child interactions when young children have disabilities. In International review of research in mental retardation (Vol. 25, pp. 35-70). Academic Press.

Steinberg, S., Liu, T., \& Lense, M.D. (2021). Musical engagement and parent-child attachment in families with young children during the Covid-19 pandemic. Frontiers in Psychology, 12.

Teggelove, K., Thompson, G., \& Tamplin, J. (2019). Supporting positive parenting practices within a community-based music therapy group program: Pilot study findings. Journal of Community Psychology, 47(4), 712-726.

Thakur, D., Martens, M. A., Smith, D. S., \& Roth, E. (2018). Williams syndrome and music: A systematic integrative review. Frontiers in Psychology, 9. https://doi.org/10.3389/fpsyg.2018.0220

Thompson, G. A., Shanahan, E. C., \& Gordon, I. (2018). The role of music-based parent-child play activities in supporting social engagement with children on the autism spectrum: A content analysis of parent interviews. Nordic Journal of Music Therapy. http://dx.doi.org/10.1080/08098131.2018.1509107

Trehub, S. E., Plantinga, J., \& Russo, F. A. (2016). Maternal vocal interactions with infants: Reciprocal visual influences. Social Development, 25(3), 665-683.

Trehub, S. E., and Gudmundsdottir, H. R. (2019). Mothers as singing mentors for infants. In Welch, G., Howard, D., and Nix, J. (Eds), The Oxford Handbook of Singing (pp. 455469). Oxford University Press. https://doi.org/10.1093/oxfordhb/9780199660773.013.25

Van Hooste, A., \& Maes, B. (2003). Family factors in the early development of children with Down syndrome. Journal of Early Intervention, 25(4), 296-309. 
Wheeler, B. L. (2018). Music Therapy Assessment: Theory, Research, and Application. Jessica Kingsley Publishers.

Williams, C. A., Driscoll, D. J., \& Dagli, A. I. (2010). Clinical and genetic aspects of Angelman syndrome. Genetics in Medicine, 12(7), 385-395.

Williams, K. E., Barrett, M. S., Welch, G. F., Abad, V., \& Broughton, M. (2015). Associations between early shared music activities in the home and later child outcomes: Findings from the Longitudinal Study of Australian Children. Early Childhood Research Quarterly, 31, 113-124. http://dx.doi.org/10.1016/j.ecresq.2015.01.004

Willinger, U., Diendorfer-Radner, G., Willnauer, R., Jörgl, G., \& Hager, V. (2005). Parenting stress and parental bonding. Behavioral Medicine, 31(2), 63-72.

Zubrick, S. R., Smith, G. J., Nicholson, J., Sanson, A., \& Jackiewicz, T. A. (2008). Parenting and families in Australia. FaHCSIA Social Policy Research Paper. 
Table 1

Demographic Characteristics by Diagnostic Group

\begin{tabular}{|c|c|c|c|c|c|c|}
\hline & $\begin{array}{l}\text { TD } \\
(n=208)\end{array}$ & $\begin{array}{l}\text { ASD } \\
(n=39)\end{array}$ & $\begin{array}{l}\mathrm{DS} \\
(n=40)\end{array}$ & $\begin{array}{l}\text { WS } \\
(n=25)\end{array}$ & $\begin{array}{l}\text { OtherDD } \\
(n=28)\end{array}$ & $F$-value/ $\chi^{2}$ \\
\hline \multicolumn{7}{|l|}{ Child } \\
\hline Sex ( $\%$ male $)$ & 46.6 & 71.8 & 47.5 & 52.0 & 46.4 & 8.64 \\
\hline Age in months (mean (SD)) & $\begin{array}{l}33.6 \\
(17.2)\end{array}$ & $\begin{array}{l}51.2 \\
(14.9)\end{array}$ & $\begin{array}{l}30.2 \\
(14.2)\end{array}$ & $\begin{array}{l}43.6 \\
(15.3)\end{array}$ & $\begin{array}{l}39.9 \\
(17.8)\end{array}$ & $12.24 * * *$ \\
\hline Only Child & 47.1 & 25.6 & 25.0 & 20.0 & 14.3 & \\
\hline \multicolumn{7}{|l|}{ Respondent } \\
\hline Relationship to child & & & & & & 4.78 \\
\hline Biological mother & 93.3 & 92.3 & 95.0 & 92.0 & 82.1 & \\
\hline Other & 6.7 & 7.7 & 5.0 & 8.0 & 17.9 & \\
\hline Education level & & & & & & $20.98^{* *}$ \\
\hline Less than Bachelor's & 10.1 & 35.9 & 17.5 & 20.8 & 21.4 & \\
\hline Bachelor's degree & 30.9 & 30.8 & 37.5 & 33.3 & 32.1 & \\
\hline Graduate degree & 58.9 & 33.3 & 45.0 & 45.8 & 46.4 & \\
\hline
\end{tabular}




\section{Table 2}

Means and Standard Deviations for the Home Music Environment and Parent-Child Affective Attachment by Diagnostic Group

\begin{tabular}{|c|c|c|c|c|c|c|}
\hline & $\mathrm{TD}$ & ASD & DS & WS & OtherDD & $F$-value \\
\hline \multirow[t]{2}{*}{ Music@Home total } & $99.3^{\circ}$ & 94.6 & $104.7^{\mathrm{a}, \mathrm{o}}$ & $100.6^{\circ}$ & $90.8^{\mathrm{t}, \mathrm{d}, \mathrm{w}}$ & $4.27 * *$ \\
\hline & $(13.0)$ & $(15.4)$ & $(10.6)$ & $(12.5)$ & $(16.4)$ & \\
\hline \multirow{2}{*}{$\begin{array}{l}\text { Child's Active Engagement } \\
\text { with Music }(\mathrm{M} @ \mathrm{H})\end{array}$} & $56.2^{\mathrm{a}, \mathrm{o}}$ & $50.3^{\mathrm{t}, \mathrm{d}, \mathrm{w}}$ & $58.3^{\mathrm{a}, \mathrm{o}}$ & $55.6^{\mathrm{a}}$ & $50.8^{\mathrm{t}, \mathrm{d}}$ & $10.49 * * *$ \\
\hline & $(6.3)$ & $(8.5)$ & $(4.8)$ & $(6.5)$ & $(8.6)$ & \\
\hline \multirow{2}{*}{$\begin{array}{l}\text { Parent Initiation of Musical } \\
\text { Activities }(\mathrm{M} @ \mathrm{H})\end{array}$} & 43.1 & 44.3 & 46.4 & 44.9 & 40.0 & 2.46 \\
\hline & $(8.7)$ & $(8.7)$ & $(7.2)$ & $(7.6)$ & $(10.9)$ & \\
\hline \multirow[t]{2}{*}{ Child music activities time } & 2.6 & 2.5 & 2.8 & 2.8 & 2.1 & 2.49 \\
\hline & $(1.0)$ & $(1.2)$ & $(1.2)$ & $(0.9)$ & $(0.6)$ & \\
\hline \multirow{2}{*}{$\begin{array}{l}\text { Function of musical } \\
\text { activities }\end{array}$} & 7.6 & 7.9 & 8.1 & 8.6 & 7.2 & 1.89 \\
\hline & $(2.2)$ & $(2.6)$ & $(1.9)$ & $(1.6)$ & $(2.7)$ & \\
\hline \multirow{2}{*}{$\begin{array}{l}\text { Parent-child affective } \\
\text { attachment }\end{array}$} & $79.9^{\circ}$ & 77.5 & $82.6^{\mathrm{a}, \mathrm{o}}$ & 79.8 & $74.8^{\mathrm{t}, \mathrm{d}}$ & $4.49 * *$ \\
\hline & $(7.7)$ & $(8.1)$ & $(6.6)$ & $(5.7)$ & $(9.5)$ & \\
\hline
\end{tabular}

$* * p<.01, * * * p<.001$ All analyses control for child age and parent education.

$\mathrm{t}=$ significantly different from TD group

$\mathrm{a}=$ significantly different from ASD group

$\mathrm{d}=$ significantly different from DS group

$\mathrm{W}=$ significantly different from WS group

$\mathrm{o}=$ significantly different from OtherDD group 
Table 3

Proportion of Participants that Endorsed Each Function of Music by Diagnostic Group

\begin{tabular}{lccccr}
\hline & TD & ASD & DS & WS & OtherDD \\
\hline Interact in social games & .86 & .89 & .97 & .87 & .85 \\
Soothe child when upset & .82 & .78 & .89 & .96 & .85 \\
Help transition between activities & .60 & .72 & .60 & .91 & .77 \\
Keep busy/pass time while waiting & .92 & .94 & .97 & .91 & .81 \\
Distract child & .75 & .78 & .91 & .96 & .81 \\
Get child's attention & .61 & .64 & .57 & .91 & .62 \\
Practice communication skills & .71 & .81 & .77 & .74 & .52 \\
Part of nighttime routine & .71 & .75 & .80 & .65 & .69 \\
Part of other routines & .80 & .81 & .86 & .87 & .65 \\
Practice academic skills & .79 & .81 & .83 & .91 & .62 \\
\hline
\end{tabular}




\section{Table 4}

Correlations Between Parent-Child Affective Attachment, Parenting Variables, Parent-Child Music and Non-Music Activities, and Child Age

\begin{tabular}{lccccc}
\hline & 1 & 2 & 3 & 4 & 5 \\
\hline 1. Attachment & & & & & \\
2. Parent self-efficacy & $.52^{* * *}$ & & & & \\
3. Parent distress & $-.50^{* * *}$ & $-.37 * * *$ & & & \\
4. Music@Home total & $.35^{* * *}$ & .11 & -.15 & & \\
5. Child age & $-.15^{* *}$ & -.06 & .11 & $-.18^{* *}$ & \\
6. Non-Musical Family Activities & $.19 * * *$ & .08 & -.06 & $.26 * * *$ & .00 \\
$* * *<01, * * * p<.001$ & & & & &
\end{tabular}


Table 5

Model Examining Parent-Child Affective Attachment

\begin{tabular}{llc}
\hline & $F$-value & $\mathrm{\eta p}^{2}$ \\
\hline Child age & 4.099 & .015 \\
Parent distress & $46.263^{* * *}$ & .143 \\
Parent self-efficacy & $71.810^{* * *}$ & .205 \\
Music@Home total score & $11.868^{* * *}$ & .041 \\
Parent education & $27.290^{* * *}$ & .089 \\
Non-musical family activities & 4.665 & .017 \\
Child diagnosis & 2.938 & .041 \\
$R^{2}$ & & \\
$* * * p<.001$ & .502 &
\end{tabular}

